\title{
Geometrical optimization of a longitudinal resonant photoacoustic cell for sensitive and fast trace gas detection
}

\author{
F. G. C. Bijnen, J. Reuss, and F. J. M. Harren ${ }^{\text {a) }}$ \\ Department of Molecular and Laser Physics, University of Nijmegen, Toernooiveld, 6525 ED Nijmegen, \\ the Netherlands
}

(Received 29 September 1995; accepted for publication 28 March 1996)

\begin{abstract}
We present a quantitative discussion of the acoustic transmission line theory pertaining to experimental results from a resonant photoacoustic cell excited in its first longitudinal mode. Window absorption is optimally suppressed by buffer volumes and tunable air columns. The acoustic behavior of an ultrasensitive one inch condenser microphone is quantitatively described. A small and sensitive photoacoustic cell has been developed for intracavity use in a $\mathrm{CO}_{2}$ waveguide laser permitting measurements of ethylene down to $6 \mathrm{pptv}$ (long term stability $20 \mathrm{pptv}$ ) with a time response of $2 \mathrm{~s}$ at a trace gas flow of $61 / \mathrm{h}$. To demonstrate the fast time response within a biological application the instant ethylene release of a single tomato is measured. (C) 1996 American Institute of Physics. [S0034-6748(96)02307-6]
\end{abstract}

\section{INTRODUCTION}

During the past decade the development of sensitive trace gas detection techniques has become more important in fields of research like environmental sensing, medicine, and biology. ${ }^{1,2}$ In the latter case, the need for sensitive trace detection concurs with the desire to perform nonintrusive measurements. Trace gas emission by plants, animals and human beings gives insight into their physiology. Trace amounts of ethylene produced by plant material are our interest since it is a hormone; the gas is measured in a continuous flow system avoiding accumulation around the tissue. ${ }^{3}$

Photoacoustic (PA) spectroscopy in combination with laser excitation forms a very sensitive detection technique offering the possibility to work with a continuous flow sampling system. ${ }^{4}$ The PA effect is based upon energy conversion from light to sound energy. In the past, many authors discussed the theoretical acoustic behavior of nonresonant and resonant cells, the latter excited either in a radial,$^{5}$ an azimuthal or a longitudinal ${ }^{6,7}$ mode. Different resonant cell types have been compared. ${ }^{8,9}$ The PA resonator excited in its first longitudinal mode and described here can be understood very well as the acoustic analogue of an electric transmission line. ${ }^{10}$

Quantitative results of the acoustic transmission line theory are presented. Within this approach, the geometry of the PA cell can be optimized for a maximum ratio of acoustic pressure amplitude (from bulk absorption) to PA background signal (from window absorption), at the same time realizing a minimum volume of the PA cell. For trace gas detection, it is important to have a low and stable background signal, otherwise additional noise is introduced, resulting in a deterioration of the detection limit of the PA system. Low background signals are especially important in an intracavity setup; due to the increased laser power, the background signal is augmented correspondingly. A small volume of the acoustic cell should be desirable to obtain a

\footnotetext{
a) Author to whom correspondence should be addressed; Electronic mail: fransh@sci.kun.nl
}

fast response of the cell in a flow-through system at low flow rates. A low gas flow is important when small gas samples (e.g., derived from small biological systems) are to be analyzed. A high flow velocity would increase the acoustic noise level and dilute the sample.

The validity of the theoretical modeling of the PA signal generation will be tested and compared with experimental results derived from an extracavity PA cell using a $\mathrm{CO}_{2}$ waveguide laser as a light source. From this, optimal size and geometry of the PA cell will be derived. In an intracavity $\mathrm{CO}_{2}$ laser arrangement, the fast time response is exploited in a biological application by measuring abrupt ethylene release of a single tomato.

\section{ACOUSTIC TRANSMISSION LINE MODEL}

A modulated heat source $H(\mathbf{r}, t)$ induces periodical changes of temperature $t$ and pressure $p$. We assume these changes to be small as compared to the average temperature $T$ and total pressure $P$. The following differential equation applies to the case without any thermal and viscous losses: ${ }^{11}$

$$
\nabla^{2} p-\frac{1}{c^{2}} \frac{\partial^{2} p}{\partial t^{2}}=-\frac{(\gamma-1)}{c^{2}} \frac{\partial H(\mathbf{r}, t)}{\partial t}
$$

with $c$ the velocity of sound $(\mathrm{m} / \mathrm{s}), \gamma=c_{p} / c_{v}$ the specific heat ratio of the gas and $H(\mathbf{r}, t)=\alpha P_{a_{g}} P_{L}(\mathbf{r}) e^{i \omega t} / S$ the heat added per unit volume. $\alpha$ is the absorption coefficient of the gas $\left(\mathrm{atm}^{-1} \mathrm{~cm}^{-1}\right), P_{a g}$ the partial pressure of the gas (atm), $P_{L}(\mathbf{r})$ the not-modulated laser power $(\mathrm{W}), S$ the cross sectional area of the duct $\left(\mathrm{m}^{2}\right)$, and $\omega=2 \pi \nu$ the angular modulation frequency.

At the wall of the resonator, losses occur due to viscosity and thermal conduction. We neglect heat conduction and viscous losses in the volume of the gas; the acoustic power loss from these effects is very small. Surface losses can be calculated from the thermal and viscous boundary layers of the gas near the duct wall. The thickness of these boundary layers are ${ }^{11}$ 
TABLE I. Transmission line parameters per unit length: $D$ is the perimeter and $S$ the cross sectional area of the duct, $P_{a_{g}}$ is the partial pressure of the gas, $P_{L}$ is the not modulated power of the incident laser beam, and $c$ is the velocity of sound. The other parameters are explained in the text.

\begin{tabular}{ll}
\hline \hline Resistance (viscous losses) & $R=\rho \omega \delta_{\nu} D / 2 S^{2}$ \\
Inductance (kinetic energy) & $\omega L=\omega \rho / S$ \\
Conductance (heat conduction losses) & $G=(\gamma-1) \omega \delta_{\kappa} D / 2 \rho c^{2}$ \\
Susceptance (potential energy) & $\omega C=\omega S / \rho c^{2}$ \\
Impedance & $Z=R+i \omega L$ \\
Admittance & $Y=G+i \omega C$ \\
Source term (absorbed laser power) & $V_{0}=(\gamma-1) P_{a_{g}} P_{L} \alpha / i \omega S$ \\
\hline \hline
\end{tabular}

$$
\delta_{\kappa}=\sqrt{\frac{2 \kappa}{\rho \omega c_{p}}}, \quad \delta_{\nu}=\sqrt{\frac{2 \nu}{\rho \omega}}
$$

with $\kappa$ the heat conductivity, $\rho$ the mean gas density, $c_{p}$ the heat capacity per unity mass, and $\nu$ the dynamic viscosity of the gas. At atmospheric pressure and audio frequencies, both boundary layers are only a fraction of a millimeter thick. For the lossless case Eq. (1) yields good solutions $p(\mathbf{r}, t)$ that describe approximately also the low-loss case. The differential equation is not valid for capillary tubes with a small diameter $\left(2 r_{\text {res }} \approx \delta_{\kappa}, \delta_{v}\right)$ (Ref. 12) and for gases with exceptionally high viscosity or heat conductivity. A uniform heat deposition over the cross section of the duct (radius $r$ ) is assumed since only longitudinal resonances (along direction $x)$ are considered. Neglecting the first term of Eq. (1) the modulated pressure $p_{0}(x, r, t)$ yields the source term:

$$
p_{0}(x, t)=\frac{(\gamma-1) \alpha P_{a g} P_{L}(x) e^{i \omega t}}{i \omega S}
$$

If an acoustic wave is transmitted through a tube, on can express the acoustic behavior with the one-dimensional electrical transmission line analogue. The basis of this theory has been described, ${ }^{11}$ extended with a matrix formalism and applied before in this field. ${ }^{10}$ Each matrix describes the characteristics of the acoustic wave in a uniform part of the cell. Coupling these matrices permits a quantitative description of the PA signal at any position in the cell with an arbitrary modulation frequency.

Within the transmission line description, properties of ducts forming a PA cell can be expressed as discrete circuit immittances per unit length, such as resistance (real part of impedance), reactance (imaginary part of impedance), conductance (real part of admittance) and susceptance (imaginary part of admittance); see Table I. These immittances assume piecewise constant values. Introducing a source of sound due to trace gas absorption of modulated laser energy yields periodical and spatially dependent changes of pressure $p_{0}(x, t)=p(x) e^{i \omega t}$. The electric analogue of the sound pressure $p(x)$ is the voltage $V(x)$; the analogue of the total flux of the gas $S u(x)$ (cross section $S$ times velocity $u$ ) is the electric current $I(x)$. The immittances reflect the energy contents (due to movement and compression) and the losses (viscous and thermal).

The differential equations for the waves in a duct in the description of the electrical transmission line are given by

$$
\frac{d V(x)}{d x}=-Z I(x), \quad \frac{d I(x)}{d x}=-V(x) Y+V_{0} Y .
$$

Expression for the impedance $Z$, the admittance $Y$ and $V_{0}$ are given in Table I. $V_{0}$ represents the time independent voltage analogue of pressure [Eq. (3)]. The solution of the differential equations are

$$
V(x)=V_{0}-Z_{c}\left(A e^{\beta x}-B e^{-\beta x}\right), \quad I(x)=A e^{\beta x}+B e^{-\beta x}
$$

with $\beta=\sqrt{Z Y}$ the propagation constant and $Z_{c}=\sqrt{Z / Y}$ the characteristic impedance of the transmission line. The amplitudes $A$ and $B$ are determined by the continuity of gas flux $I(x)$ and the pressure $V(x)$ at the boundaries between two connected ducts.

In the case of large changes in radii between the resonator and the larger buffer, the one-dimensional transmission line approach is only partially valid. From the expression for a small opening inside a large duct, an impedance condition at the interface can be deduced. ${ }^{11}$ To adjust the transmission line model for these effects, additional immittances $Z_{\mathrm{ct}}=R_{\mathrm{ct}}+i \omega L_{\mathrm{ct}}$ (ct: corrected transmission line) should be implemented at the junction between two connected ducts with:

$$
L_{\mathrm{ct}}=\frac{\rho}{4 r_{\text {res }}}, \quad R_{\mathrm{ct}}=\frac{\sqrt{2 \rho \omega \nu}}{8 S_{\text {res }}} \ln \left(\frac{2 r_{\text {res }}}{\delta_{\nu}}\right) .
$$

A more thorough derivation of coupling between two ducts is described elsewhere. ${ }^{15}$

At the ends of the cell, heat flux can be introduced due to laser power absorption by the windows. Air layers, with a thickness of approximately $\delta_{\kappa}$, are heated by the windows resulting in a flux introduced at these points given by ${ }^{14}$

$$
I_{w}=\frac{\delta_{\kappa} \alpha_{w} \mu_{w}^{2} P_{L}}{T \kappa_{w}}
$$

with $\mu_{w}=\sqrt{2 D_{w} / \omega}$ the diffusion length in the window, $D_{w}$ the thermal diffusivity, $\alpha_{w}$ the absorption coefficient, and $\kappa_{w}$ the heat conductivity of the window: $T$ is the ambient temperature.

Large condenser microphones or air columns attached to the transmission line of the detector can change the acoustic behavior considerably. The input impedance of these lumped elements need therefore to be determined. In the Appendix, the input impedance $Z_{\text {mic }}$ of a condenser microphone with a small volume in front of the membrane is derived.

Another lumped element can be a tunable air column (tac Fig. 1) of length $l_{\text {tac }}$ attached to the cell volume on one side and closed at the other. The represented impedance $Z_{\mathrm{tac}}=V_{\mathrm{tac}} / I_{\mathrm{tac}}$ in the line can be described by a piece of transmission line of length $l_{\text {tac }}$ with the condition of zero current at the closed end of the tube $I_{\mathrm{tac}}\left(l_{\mathrm{tac}}\right)=0$; therefore $\left.Z_{\text {tac }}\left(l_{\text {tac }}\right)=\infty\right)$ and without a voltage source in the column $\left(V_{0}=0\right)$. Then from Eq. (5) follows:

$$
Z_{\mathrm{tac}}=Z_{c_{\mathrm{tac}}} \frac{1+e^{2 \beta_{\mathrm{tac}} l_{\mathrm{tac}}}}{1-e^{2 \beta_{\mathrm{tac}} l_{\mathrm{tac}}}} .
$$




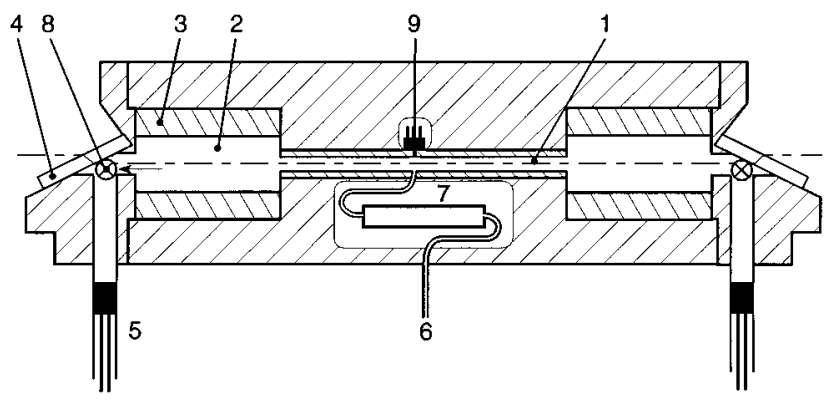

FIG. 1. Resonant photoacoustic cell. (1) Resonator; (2) buffer volume (maximum diameter 40, length $50 \mathrm{~mm}$ ); (3) buffer ring to decrease buffer radius; (4) ZnSe Brewster window; (5) tunable air column; (6) inlet gas flow; (7) $\lambda / 4$ notch filter; (8) outlet gas flow; (9) Knowless microphone.

\section{EXPERIMENT}

\section{A. General experimental arrangement}

Several cell designs have been tested and compared with theory. The influence of the cell geometry on the PA signal was investigated employing a tunable IR $\mathrm{CO}_{2}$ waveguide laser with an output power of $2 \mathrm{~W}$. The aim of this research was to employ the cell in an intracavity arrangement. A number of the experiments were performed extra cavity with the laser beam focused through the PA cell by a curved mirror $(f=500 \mathrm{~mm}$; beam waist $=1 \mathrm{~mm})$ tilted under a angle of about $30^{\circ}$ : the waist was small enough to avoid wall absorptions. A chopper (blade 50\% open) modulated the laser beam, resulting in a square wave modulation. The PA signals were monitored by means of a lock-in amplifier; the laser power was monitored behind the PA cell by a power meter containing a thermopile.

The PA cell consisted of a polished brass resonator $\left(l_{\text {res }}=100 \mathrm{~mm}, r_{\text {res }}=3 \mathrm{~mm}\right)$ inside an aluminum housing (Fig. 1). A small resonator radius was preferred since this yields a higher cell constant; the cell constant $F$, the quality factor $Q$ and the resonance frequency $\omega$ of a fully open resonator (=or: no buffer volumes) are given by ${ }^{13}$

$$
F_{\text {or }}=\frac{G(\gamma-1) l_{\text {res }} Q_{\text {or }}}{2 \pi r_{\text {res }} l_{\text {res }} \omega_{\text {or }}} \propto \frac{\sqrt{l_{\text {res }}}}{r_{\text {res }}}, \quad Q_{\text {or }} \propto \frac{r_{\text {res }}}{\sqrt{l_{\text {res }}}}, \quad \omega_{\text {or }}=\frac{\pi c}{l_{\text {res }}}
$$

with $G \approx 1$ determined by the geometry of the cell. An even smaller radius of $2 \mathrm{~mm}$ was tested but gave rise to high PA background signals during intracavity operation due to absorption of the wings of the Gaussian laser beam profile. The cell had two $\mathrm{ZnSe}$ windows at Brewster angle. The length of the cylindrical holes drilled into the aluminum block to connect the buffer volume with the window was minimized to avoid additional resonances.

The experiments were performed with two types of microphones. First a small Knowless EK3024 electret microphone (sensitivity $22 \mathrm{mV} / \mathrm{Pa}$ at $1600 \mathrm{~Hz}$ ), electrical noise 40 $\mathrm{nV} / \mathrm{Hz}^{1 / 2}$ ) was employed to investigate the acoustical performance on changing buffer dimensions of the cell. No influence of this microphone on the acoustic performance of the cell was observed (an additional microphone did not change the acoustical properties of the cell). To reach lower detection limits and to study the influence on the acoustic behav-
TABLE II. Properties of $\mathrm{ZnSe}$ at room temperature $(T=295 \mathrm{~K})$.

\begin{tabular}{ll}
\hline \hline Heat conductivity $\kappa_{s}$ & $18 \mathrm{~W} / \mathrm{m} \mathrm{K}$ \\
Thermal diffusivity $D_{s}$ & $1.005 \times 10^{-5} \mathrm{~m}^{2} / \mathrm{s}$ \\
Absorption coefficient $\alpha_{s}$ & $0.002 \mathrm{~cm}^{-1}$ \\
\hline \hline
\end{tabular}

ior, a large 1 in. condenser microphone was introduced (Bruel and Kjaer 4179, sensitivity $1 \mathrm{~V} / \mathrm{Pa}$ electrical noise $\left.200 \mathrm{nV} / \mathrm{Hz}^{1 / 2}\right)$. One should avoid acoustic leaks from not coupling properly the microphone to the resonator; it causes a drastic reduction of the signal amplitude and quality factor. The microphones were therefore glued (Knowless) or sealed with $o$-rings $(\mathrm{B} \& \mathrm{~K})$ to the resonator.

To test the response of a cell for trace amounts of gas, a calibrated mixture of 1.2 ppmv $\mathrm{C}_{2} \mathrm{H}_{4}$ in $\mathrm{N}_{2}$ was flowed through the cell at STP. The modeling experiments for the window signals were performed with pure nitrogen ${ }^{16}$ and one blackened window. Calculations for the gas absorption signal included no signal generated at the windows. For window signal absorption, Eq. (7) was included in the model while no gas absorption was considered $\left(V_{0}=0\right)$. The physical properties of $\mathrm{ZnSe}$ are shown in Table II and was used in the model. The pressure amplitude response of the PA cell due to gas absorption is expressed by the cell constant $\mathrm{F}$ $(\mathrm{Pa} \cdot \mathrm{cm} / \mathrm{W})$. Since $F$ is independent of the amount of absorbed energy, the experimentally determined cell constant $F_{\text {exp }}$ was obtained by correcting the measured signal amplitude for the absorption strength of the trace gas, the concentration, the laser power, and the microphone sensitivity:

$$
F_{\exp }=\frac{\pi A_{m}}{4 S_{m} \alpha P_{a_{g}} P_{L}} .
$$

With $A_{m}$ the peak-peak signal of the microphone in $(V)$ and $S_{m}$ denote the sensitivity of the microphone (V/Pa). The factor $4 / \pi$ reflects the Fourier transform value of the square wave power modulation. For ethylene at the $10 \mathrm{P} 14 \mathrm{CO}_{2}$ laser line $\alpha=30.4\left(\mathrm{~atm}^{-1} \mathrm{~cm}^{-1}\right){ }^{17}$ To compare experimental and theoretical cell constants $F_{\text {exp }}$ was corrected for the ratio theoretical/experimental $Q$ value.

\section{B. Influence of buffer volumes}

To study the validity of the transmission line model developed above and to determine the critical parameters involved, the dimensions of the buffers were varied and compared to the calculation. In a computer program based upon the presented theory, the PA cell consisted of five subcells: the middle subcell represents the resonator $\left(l_{\text {res }}, r_{\text {res }}\right)$, at both sides connected to two buffers $\left(l_{\text {buf }}, r_{\text {buf }}\right)$; the last two subcells are the connections between buffer and window. Here, the tunable columns near the window were set at zero length and were not taken into account. In the PA cell, the cross section of the subcell connecting buffer and window varied since the windows were positioned under the Brewster angle. In the model, the length for these tubes was taken as the total volume divided by the cross section of the pipe at the connection to the buffer. This is a proper approximation if the length is much smaller then the acoustic wavelength. 

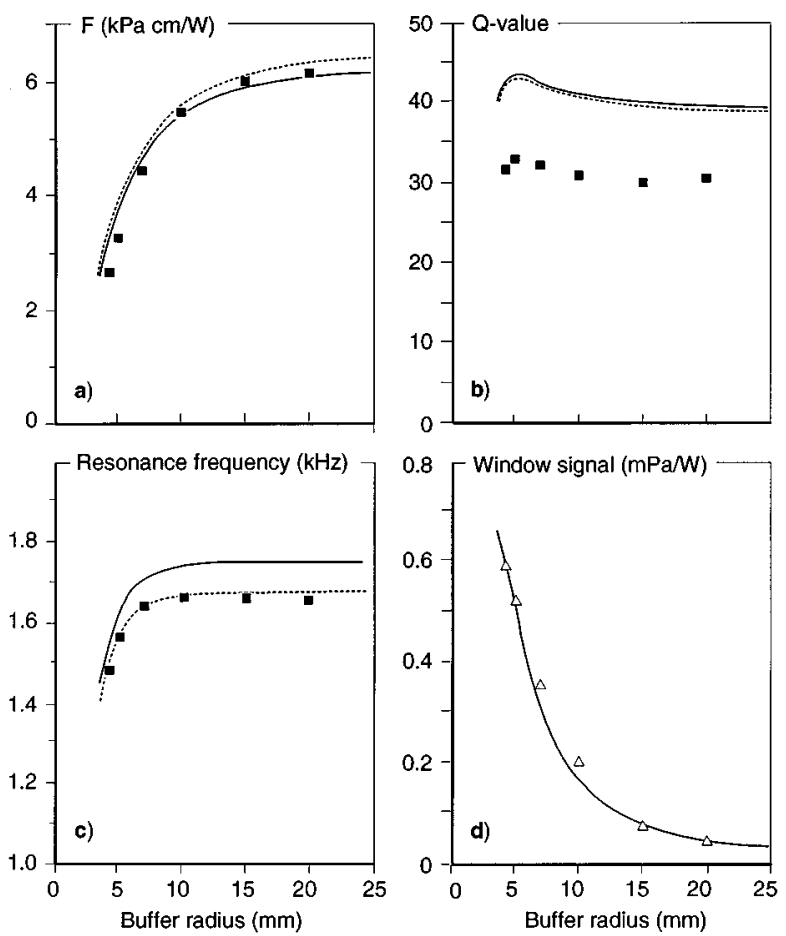

FIG. 2. Cell constant (a), quality factor (b), and resonance frequency (c) of the photoacoustic gas signal for various buffer radii of the standard (-) and corrected $(\cdots)$ transmission line model and the experimental data $(\boldsymbol{\square})$. (d) Photoacoustic $\mathrm{ZnSe}$ window absorption signal $\left(\alpha_{w}=0.002 \mathrm{~cm}^{-1}\right)$ : corrected transmission line model $(-)$ and experiment $(\triangle$, black window, values scaled to theory). Other parameters: $r_{\text {res }}=3 \mathrm{~mm}, l_{\text {res }}=100 \mathrm{~mm}$, and $l_{\text {buf }}=50$ $\mathrm{mm}$.

The inner diameter of the buffer volume was reduced by aluminum rings of various inner diameter and was sealed to the original buffer surface with $o$-rings (Fig. 1). The results for PA signal generation by gas absorption are presented in Fig. 2. Comparing the experimental resonance frequency with calculations gives an agreement within $1 \%$; if the corrected transmission line model [Eq. (7)] was not included, the agreement was only within $8 \%$. The theoretical $Q$ values are overestimated as compared to experimental results; additional losses seem to be taken into account within the model. The calculated cell constant $F$ corresponds very well with experimental values.

For comparison, the expression for the open tube [Eq. (9) $]$ yields $Q_{\text {or }}=38.5$ and $\nu_{\text {or }}=1750 \mathrm{~Hz} \quad\left(r_{\text {res }}=3 \mathrm{~mm}\right.$, $\left.l_{\text {res }}=100 \mathrm{~mm}\right)$. These values agree well with the calculations obtained by the standard transmission line model for large $l_{\text {buf }}$. The agreement allows us to determine the geometrical factor $G=1.2$, yielding a cell constant of $F_{\text {or }}=6 \times 10^{3}$ $(\mathrm{Pa} \mathrm{cm} / \mathrm{W})$.

The quality factor (Fig. 2) showed a maximum at $r_{\text {buf }}=2 r_{\text {res }}$. This can be understood as follows: at large $r_{\text {buf }}$ the acoustic standing wave is mainly confined to the resonator area. The losses are determined by the resonator. At smaller radii $\left(r_{\text {buf }} \approx 2 r_{\text {res }}\right)$ a better coupling between resonator and buffer distributes the energy of the standing wave over both. Since $r_{\text {buf }} \geqslant r_{\text {res }}$ and $Q \propto r / \sqrt{ } l$ the quality factor increases. At smaller $r_{\text {buf }}$, the ratio of acoustic volume over

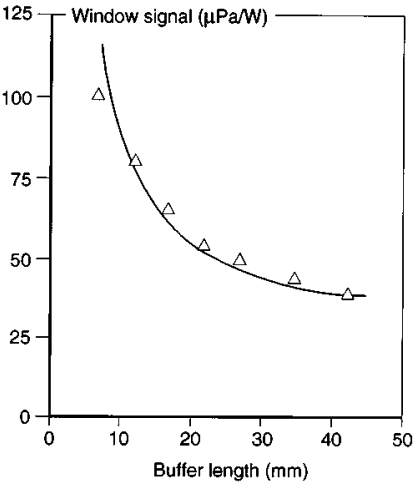

FIG. 3. Comparison between experimental and calculated PA ZnSe window absorption signal $\left(\alpha_{w}=0.002 \mathrm{~cm}^{-1}\right)$ for various buffer lengths: corrected transmission line model $(-)$ and experiment $(\triangle$, tunable black piston, values scaled to theory). Other parameters: $r_{\text {res }}=3 \mathrm{~mm}, l_{\text {res }}=100 \mathrm{~mm}$, and $r_{\text {buf }}=20 \mathrm{~mm}$.

wall surface decreased resulting in a lower value for the quality factor.

The experimental results for the window signal absorption amplitude were obtained by making one window optically black to increase the signal amplitude for the modeling purpose; the beam was partially $(50 \%)$ absorbed. In this way, the phase and the relative amplitude of the window signal were determined. The experimental results were scaled to the calculations. As can be seen from Fig. 2 at small buffer radii, the ZnSe window signal became stronger $\left(\alpha_{\omega}=0.002 \mathrm{~cm}^{-1}\right)$. The window absorption signal had the same $Q$ value and resonance frequency as for the gas signal and is therefore not shown. In this way, an improvement of the gas-windowabsorption-ratio by a factor 3000 has been achieved compared to results derived elsewhere ${ }^{13}$ with a banana type longitudinal resonant cell.

To investigate the influence of the length of the buffers on the transmission of the window signal, one buffer was varied in length. At one end, the Brewster window holder was taken off and a black absorbing piston was introduced. The piston could slide along the length axis sealed with $o$-rings to the cylindrical buffer wall, thereby, varying the buffer length. In this way, the total laser radiation was absorbed resulting in a strong signal suitable for a comparison between theory and experiment. As can be seen from Fig. 3, the best attenuation was achieved at a buffer length of 50 $\mathrm{mm}(\lambda / 4)$ : the optimal length for destructive interference for pressure amplitudes introduced into the buffer from either one of the sides.

Besides the influence of the window signal, the gas absorption amplitude was tested by positioning aluminum rings inside both buffers to reduce the buffer length with (now) transparent windows. Theory and experiment agreed and indicated that the gas signal amplitude decreases drastically when $l_{\text {buf }}<\lambda / 8$; resonance frequency and quality factor, both for window and gas signal, are not much affected by changing the buffer length.

\section{Tunable air columns near the windows}

A good choice of buffer dimensions results in a weak acoustic coupling between the windows and the resonator 

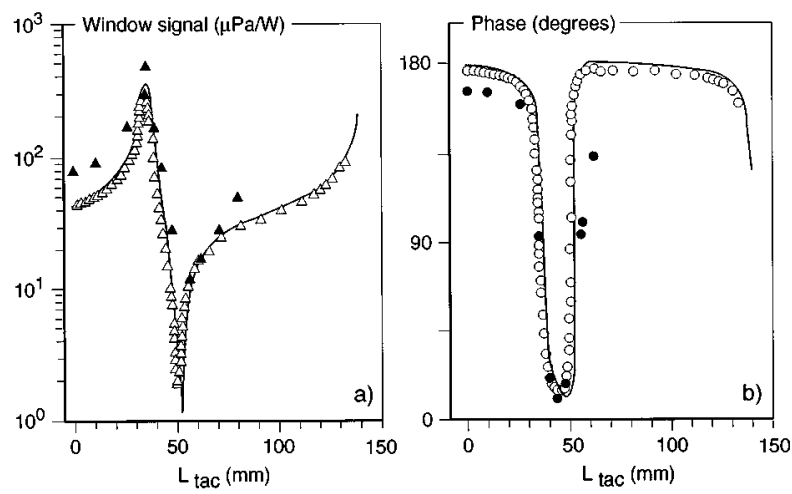

FIG. 4. PA window absorption signal (a) and phase (b) as function of $l_{\text {tac }}$ [calculation: $(-)$, experiment $(\triangle, \bigcirc)$, black window, values scaled to theory]. Other parameters: $r_{\text {res }}=3 \mathrm{~mm}, l_{\text {res }}=100 \mathrm{~mm}, r_{\text {buf }}=20 \mathrm{~mm}$, and $l_{\text {buf }}=50 \mathrm{~mm}$. Experimental data are supplemented with results for transparent $\mathrm{ZnSe}$ windows $\left(\alpha_{w}=0.002 \mathrm{~cm}^{-1}\right)$ in an intracavity setup $(\boldsymbol{\Lambda}, \mathbf{O})$. Other parameters: $r_{\text {res }}=4 \mathrm{~mm}, l_{\text {res }}=100 \mathrm{~mm}$

and yields a low window background signal. Operating the PA cell in an intracavity arrangement can still result in a significant window absorption signal due to the high laser power. Further reduction of window signal can be achieved by positioning tunable air columns close to the windows (Fig. 1). For this experiment the cell was employed with a modified brass resonator $\left(r_{\text {res }}=4 \mathrm{~mm}, l_{\text {res }}=100 \mathrm{~mm}\right.$ ) which was coated with a polished gold surface to ensure that no signal was produced due to the absorption of the resonator wall. ${ }^{18}$ The calculations and experiments were obtained for two different buffer radii: $r_{\text {buf }}=20 \mathrm{~mm}$ (Fig. 4) and $10 \mathrm{~mm}$ (not shown). Intracavity and extracavity experiments were performed: the extracavity results were obtained with one blackened window (as described above); the intracavity experiments (120 W laser power) with transparent Brewster windows to determine the absolute window signal and the actual suppression due to the tunable column.

For the calculations, only one window column was tuned in length while at the other window no window absorption signal was introduced. The radius of the columns' tubes is chosen to be equal to the radius of the tubes connecting the buffers with the windows $\left(r_{\mathrm{tac}}=5 \mathrm{~mm}\right)$. Calculations showed, however, that $r_{\text {tac }}$ is not critical.

As shown in Fig. 4, the amplitude, phase of calculations, and experimental results are in good agreement; the phase agrees even without any scaling. A steep (and deep) minimum in the window signal amplitude is observed at a length of approximately $50 \mathrm{~mm}$ which corresponds to $\lambda / 4$. As shown, a reduction of the window signal can be achieved by a factor of 20 (theoretical 30) with $r_{\text {buf }}=20 \mathrm{~mm}$; at $r_{\text {buf }}=10$ mm experimentally as well as theoretically a suppression by a factor of 50 is achieved. The position of the minimum depends on the overall geometry of the cell. Calculations show that changing the position of the tunable air column near the window result in a change in position of the minimum. Since the minimum is only a few millimeters wide, it is better to determine the position experimentally. For these investigations, PA experiments were supported by loudspeaker experiments with sound at resonance frequency entering the cell at the (former) position of the windows. Even for windowless cell design, tunable air columns seem to be a promising possibility to reduce noise. ${ }^{9}$

The results of an intracavity experiment employing a larger radius resonator $\left(r_{\text {res }}=4 \mathrm{~mm}, l_{\text {res }}=100 \mathrm{~mm}\right)$ and transparent Brewster windows are also shown in Fig. 4. One of the columns was tuned while the other was kept at a fixed length yielding minimum window signal. The signal shown was corrected for the (small) contribution of the window with the fixed column. Interchanging the function of both columns resulted in a maximum signal amplitude half of the one shown in Fig. 4. Therefore, a direct comparison between the absolute signal intensity and the calculated results in an agreement within a factor of 2 . The minimum is less pronounced as predicted by theory. This is probably due to small amounts of trace gas present in the carrier flow. An overall decrease in intracavity window signal is obtained by a factor of 8 .

The suppression of the window signal can be explained along the same lines as for the buffer length; after the acoustic wave is reflected and traveled through the column, it returns to its origin out of phase with the newly introduced wave. Since the reflected wave has lost some energy at the wall of the air column, no perfect destructive interference is accomplished. Simulation of an ideal case in which no losses at the wall of the tunable air column occur result in perfect extinction of the wave emerging from the windows to the resonator. An improvement in destructive interference can be achieved by choosing a larger radius tunable air column. A homogeneous change of temperature or a gas composition result in a different sound velocity which affects the resonance frequency but will not affect the length of the tunable air column.

\section{Influence of a one inch condenser microphone}

To test the influence of an one inch microphone onto the resonator instead of a tiny Knowless microphone, a special designed PA cell was used $\left(r_{\text {res }}=2 \mathrm{~mm}, l_{\text {res }}=100 \mathrm{~mm}\right.$, $r_{\text {buf }}=20 \mathrm{~mm}$ and $l_{\text {buf }}=50 \mathrm{~mm}$ ). The acoustic coupling of the resonator tube to the cylindrically shaped volume $\left(r_{v}=11.5\right.$ mm, Fig. 9) in front of the membrane was realized through a small cylindrical hole $\left(r_{d}=1.45 \mathrm{~mm}, l_{d}=0.7 \mathrm{~mm}\right)$. The height of the air column in front of the membrane $h_{v}$ could be adjusted by pulling the microphone away from the resonator. The influence of the column height on the resonance conditions is presented in Fig. 5. In the Appendix, the full expression for the input impedance of the B\&K 4179 microphone is presented. To demonstrate the advantage of incorporating the fully developed model, the first curve represents the volume in front of the microphone as Helmholtz resonator [Eq. (A3)]. For comparison, the values for the resonator without microphone and additional volumes are: $F=9500$ $\mathrm{Pa} \mathrm{cm} / \mathrm{W}, Q=25.8$ and $v_{\text {res }}=1748 \mathrm{~Hz}$. The second curve represents the Helmholtz resonator with microphone losses but without the viscosity losses from the air gap [Eq. (A8)]. The third curve incorporates the full expression. Additional losses should still be taken into account to explain the difference in quality factor.

The sensitivity of the B\&K 4179 microphone is superior to that of the Knowless microphone, and a satisfactory ex- 

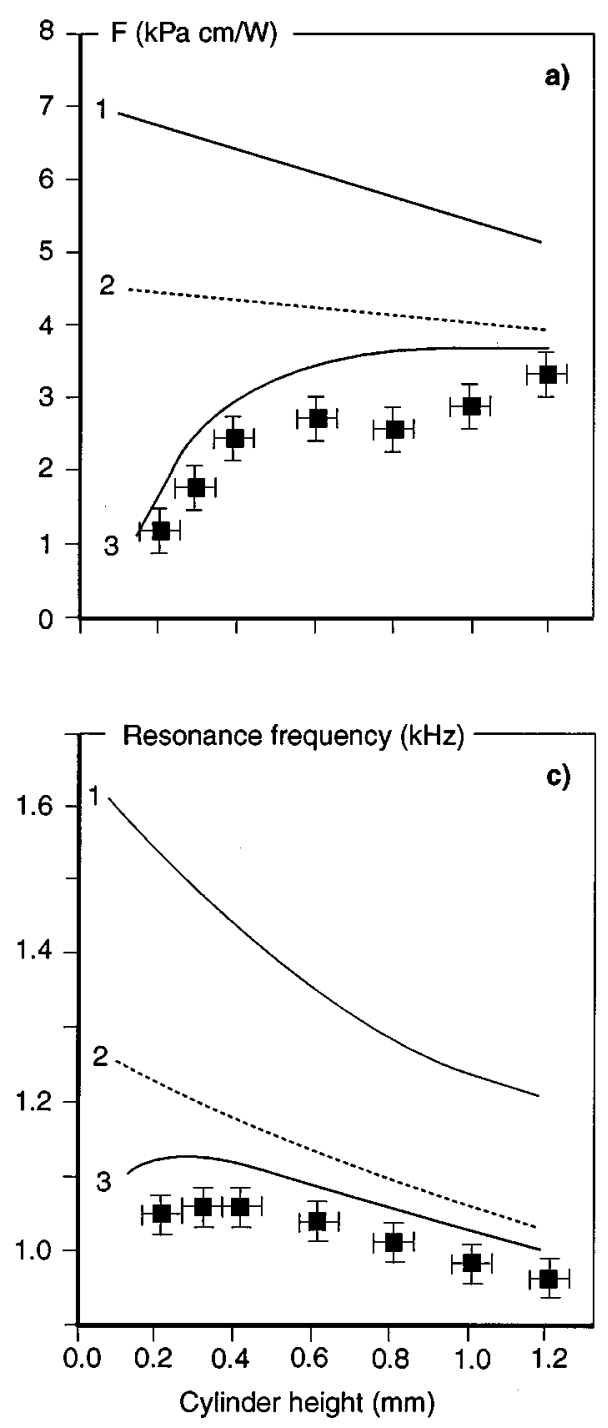

planation was found for the features it introduces in acoustic behavior. Nonetheless, this microphone was not employed in the rest of these experiments. The extremely sensitive microphone needs sufficient acoustical shielding from the laboratory noise which deteriorates the $\mathrm{S} / \mathrm{N}$ ratio. An improved design, stiffer and heavier, has been developed, Bijnen et al. ${ }^{18}$

\section{E. Trace gas inlet}

If the gas flow enters at one of the buffers volumes ${ }^{4}$ and flows through the resonator tube leaving the cell via the other buffer, a significant loss in time response results. This can be improved by entering the cell at the center of the resonator directly opposite of the microphone (Fig. 1). However, a significant increase in flow noise and interfering sound from outside the cell can then be expected. Flow noise is observed above $10 \mathrm{l} / \mathrm{h}$ which limits the minimum response time of the detector. The acoustic noise arriving from outside through the flow system can effectively been suppressed by two $\lambda / 4$ tubes in line with the trace gas flow. These $\lambda / 4$ tubes $(50 \mathrm{~mm}$ length) have alternating a large $(6.4 \mathrm{~mm})$ and a small $(0.8$ $\mathrm{mm}$ ) diameter forming an acoustic notch filter at the reso-

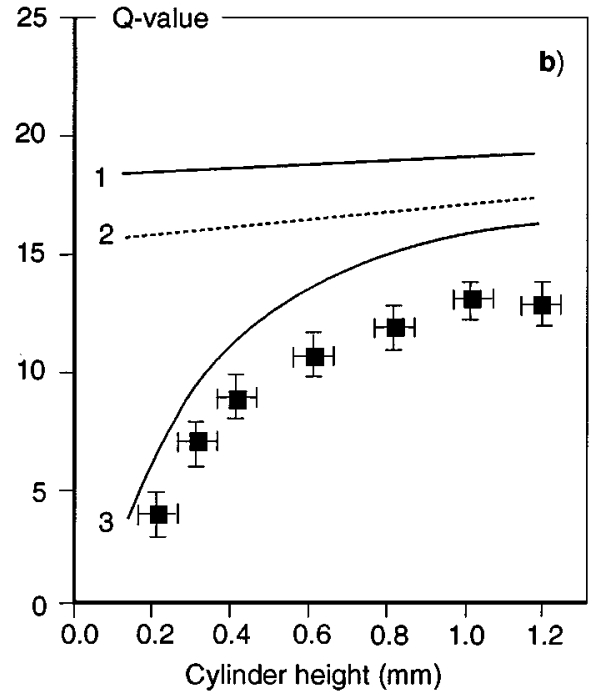

FIG. 5. Photoacoustic signal employing a condenser microphone. Cell constant (a), quality factor (b) and resonance frequency (c) are presented for varying cylinder height $h_{v}$ in front of the membrane. Line 1 represents the theoretical curve from the volume in front of the microphone acting as Helmholtz resonator [Eq. (A3)]. At line 2 the microphone losses are included but not Eq. (A8); the third curve incorporates the full expression. The experimental results are given by $\boldsymbol{\square}$. Other parameters: $r_{\text {res }}=2 \mathrm{~mm}, l_{\text {res }}=100$ $\mathrm{mm}, r_{\text {buf }}=20 \mathrm{~mm}$, and $l_{\text {buf }}=50 \mathrm{~mm}$.

nance frequency of the resonator. In our case, one damping unit sufficed to decrease the acoustic noise coming through the flow system. In our case, a suppression of a factor of 60 was found in correspondence with the model that the suppression goes quadratic with the ratio of tube radii $(6.4 / 0.8)^{2}$. However, the increased damper radii slow down the time response of the trace gas detector. If the gas inlet diameter onto the resonator is large, additional acoustical losses are introduced; therefore, we used only an entrance diameter of $0.8 \mathrm{~mm}$.

To test the time response of the detector $0.1 \mathrm{ml}$ of $\mathrm{N}_{2}$ with 1.2 ppmv ethylene was injected into a constant flow of nitrogen. At a flow of $6 \mathrm{l} / \mathrm{h}$ and no tubes in line with the trace gas flow the response time of the detector was $1.2 \mathrm{~s}$ full width at half-maximum (FWHM). Introducing one notch filter the response time increased to $2 \mathrm{~s}$.

\section{OPTIMAL DETECTOR DESIGN, A RECIPE}

Many approaches have been treated in the previous section to optimize a PA cell. Here we summarize the results and discuss the parameters to construct a small, fast and sensitive resonant PA cell. The design criteria for the geom- 
TABLE III. Photoacoustic cell design considerations.

\begin{tabular}{|c|c|}
\hline Source of trouble & Solution \\
\hline Window absorption signal & $\begin{array}{l}\text { Tunable columns at windows } \\
\text { Length buffers } \\
\text { Large radius buffer volumes }>3 r_{\text {res }}\end{array}$ \\
\hline AR window reflection & Employing Brewster windows \\
\hline $\begin{array}{l}\text { Absorption of radiation } \\
\text { at resonator wall }\end{array}$ & $\begin{array}{l}\text { Larger radius of resonator } \\
\text { Resonator material with high conduction } \\
\text { and reflection properties } \\
\text { (e.g., polished gold coated copper) }\end{array}$ \\
\hline Chopper noise & $\begin{array}{l}\text { Stabilized chopper control } \\
\text { High precision chopper blades } \\
\text { Low } Q \text {-value (with high cell constant) }\end{array}$ \\
\hline Gas sample flow noise & Reducing flow rate $<10 \mathrm{l} / \mathrm{h}$ \\
\hline Laboratory noise & $\begin{array}{l}\text { Notch filter in sample flow } \\
\text { Heavy mass cell construction } \\
\text { Acoustic shielding } \\
\text { Tunable columns at windows } \\
\text { Vibration damped mounting }\end{array}$ \\
\hline
\end{tabular}

etry of the cell depend on the interfering sources which lower the detection limit; some of these background and noise sources are listed in Table III. For the longitudinal resonant cell, we cannot simply deduce design criteria from the formulae in the transmission line model. Matrix calculations are needed to describe the acoustical behavior of the cell.

By changing the radii and lengths of the buffers and resonator, we can deduce some simple approximate formulas. For the dimensions of the buffer volume, we only consider the case where the length of the buffer is optimal for window signal suppression, i.e., $l_{\text {res }}=2 l_{\text {buf }}\left(l_{\text {res }} \gg r_{\text {res }}\right)$. The dependence of the gas absorption $p_{g}$ and the window absorp-
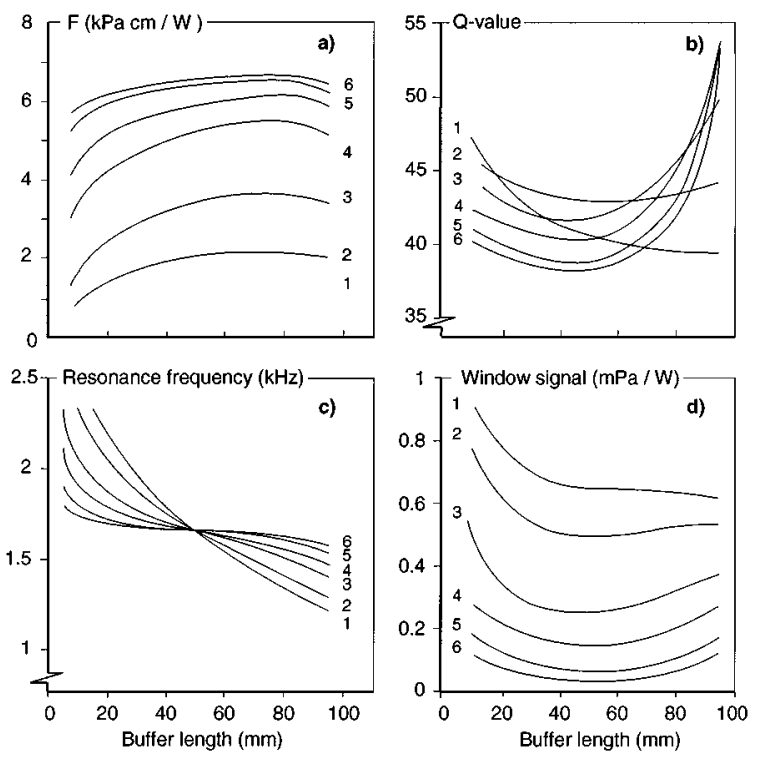

FIG. 6. Theoretical graphs of acoustic response on varying buffer parameters $\left(r_{\text {res }}=3 \mathrm{~mm}, l_{\text {res }}=100 \mathrm{~mm}\right.$ ) giving cell constant (a), quality factor (b), resonance frequency (c), and window absorption signal (d). Buffer radii of $4,5,7.5,10,15$ and $20 \mathrm{~mm}$ correspond respectively to curves $\mathrm{nr} 1,2,3,4$, 5 and 6.

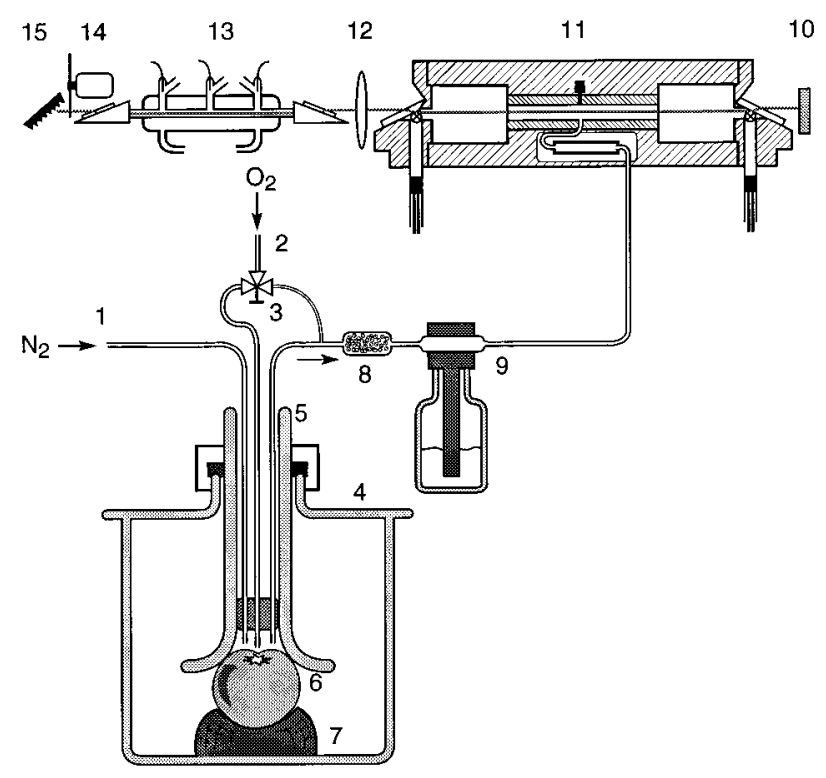

FIG. 7. Intracavity $\mathrm{PA} \mathrm{CO}_{2}$ waveguide laser arrangement with a scheme of the gas flow for performing the measurements on cherry tomatoes. (1) $\mathrm{N}_{2}$ inlet; (2) $\mathrm{O}_{2}$ inlet; (3) switching valve; (4) cuvette; (5) insert to keep tomato at its place; (6) tomato; (7) pressed foam; (8) $\mathrm{KOH}$ scrubber; (9) cooling trap; (10) end mirror; (11) photoacoustic cell; (12) intracavity lens $(f=250$ $\mathrm{mm}$ ); (13) $\mathrm{CO}_{2}$ discharge tube; (14) chopper; (15) grating.

tion pressure amplitude $p_{w}$ on the ratio of buffer and resonator radii is (see in Fig. 6)

$$
p_{g} \propto \frac{\sqrt{l_{\text {res }}}}{r_{\text {res }}}\left[1-\frac{r_{\text {res }}^{2}}{r_{\text {buf }}^{2}}\right], \quad p_{w} \propto \frac{r_{\text {res }}}{r_{\text {buf }} \sqrt{l_{\text {res }}}} .
$$

The ratio between the gas absorption signal and window signal becomes then

$$
\frac{p_{g}}{p_{w}} \propto\left[\frac{r_{\text {buf }}}{r_{\text {res }}}\right]^{2} l_{\text {res }} .
$$

From Fig. 6, it can be seen that the optimal buffer length is $\lambda / 4$. In that case optimal window signal suppression is achieved. Choosing a buffer length of $\lambda / 8$ has the advantage of a shorter cell and, although not optimal, a good suppression of window signal is still possible. If no problems concerning buffer volume or overall size have to be considered, the radii of the buffers should be large for optimal operation. A practical radius can be deduced of $r_{\text {buf }} \approx 3 r_{\text {res }}$. Above this value gas absorption amplitude is barely influenced.

A small radius for the resonator of the resonant cell is advantageous since a larger gas absorption signal is achieved. The limit for the radius of the resonator is mainly determined by the wings of Gaussian laser beam profile hitting the wall; $r_{\text {res }}$ should roughly be three times the waste to reduce wall absorption to an acceptable level.

To distinguish gas absorption signal from other (wall, window, interfering gas) signals, one has to switch the $\mathrm{CO}_{2}$ laser to other laser lines. In a simple calculation, the specific gas absorption signal and the background signals can be deduced. However, repositioning of the laser beam to its original wavelength can give a different configuration in the laser cavity (deviation in grating position, thermal drift) and might 
cause irreproducible absorption signals. In this way, the background signal becomes unstable affecting the detection limit. In an intracavity arrangement, one should be careful not to choose the resonator radius too small. The advantage of small resonator is lost due to a large and unstable background signal.

The length of the resonator determines the resonance frequency. If the sensitivity of the detector would be limited by fundamental noise sources, a resonance frequency around $1 \mathrm{kHz}$ would be optimal. Below $1 \mathrm{kHz}$, the $1 / f$ amplifier noise is the main source. Above $1 \mathrm{kHz}$ the frequency independent Brownian noise takes over. Since the pressure amplitude is inversely proportional to the square root of the resonance frequency [Eq. (11)], a preferable resonance can be found between 500 and $1500 \mathrm{~Hz}$. This limits the choice (in the case of air) to a length of $100-300 \mathrm{~mm}$. If the only argument would be optimal signal enhancement, the choice for a large $(300 \mathrm{~mm})$ resonator is advantageous $\left(p_{g} \propto \sqrt{l_{\text {res }}}\right)$. Shorter resonator lengths are necessary in the case of an intracavity setup due to the limited space inside the cavity. Also, the fast time response of the cell demands a small (i.e., short) cell.

Especially in an intracavity setup, the window signal contribution can be limiting for the sensitivity of the detector. One might choose to put tunable columns at the windows as an alternative for large $r_{\text {buf. }}$. The gas absorption signal is not influenced, whereas, the window absorption signal can be suppressed using the proper $\lambda / 4$ wavelength. Since the exact optimal length of these columns depends on the total geometry of the cell, it is advisable to optimize the length when the cell is operated in the setup. By tuning the window columns, they can additionally serve to distinguish between different background sources of the PA cell. When other sources of background signal (e.g., small amounts of trace gas or resonator wall absorption) are observed, they do not allow a signal dip for $l_{\text {tac }} \approx \lambda / 4$. Tuning the column with transparent windows, we observed a more shallow minimum.

The choice for Brewster windows in stead of antireflection (AR) coated windows was based upon the experience that the latter reflect radiation. In intracavity operation, this backreflection resulted into instability of the PA signal. Introduction of a small angle for the AR windows solved the problem of instability; however, the PA background signal increased due to larger reflection toward the buffers and resonator. The application of AR windows, in contrast to Brewster windows, is therefore restricted.

The microphone choice depends on the amount of acoustic noise arriving at the microphone. Using a very sensitive microphone possesses a stiff and heavy PA cell, employing materials like stainless steel in stead of aluminum. In our first aluminum cells, there was no use to operate the sensitive $\mathrm{B} \& \mathrm{~K} 4179$ microphone since the noise arriving from the lab (50 $\mu \mathrm{Pa} / \mathrm{Hz}^{1 / 2}$ was larger than the pressure equivalent of the electrical noise of a Knowless electret microphone (2 $\left.\mu \mathrm{Pa} / \mathrm{Hz}^{1 / 2}\right)$. As described elsewhere, ${ }^{18}$ the $\mathrm{B} \& \mathrm{~K}$ microphone has operated successfully in a better constructed cell. If one applies a sensitive condenser microphone, the height of the air column in front of the membrane should at least be $1 \mathrm{~mm}$ deep to avoid loss of signal (Fig. 5). Attention should be paid to the cross section of the connection between resonator and microphone: its diameter should be large to avoid viscous losses while its length should be short $(<5 \mathrm{~mm})$. The performance of the cell was strongly influenced by the B\&K condenser microphone resulting in a downshift of the resonance frequency by $500 \mathrm{~Hz}$ (Fig. 5). For resonators with a larger volume, this influence is less pronounced; e.g., only a $60 \mathrm{~Hz}$ downward shift was found with a larger resonator $(15 \mathrm{~mm}$ diam, $150 \mathrm{~mm} \mathrm{length}^{18}$ ).

Admitting the flow at the center of the resonator has the advantage of a fast time response. Care should be taken with the diameter of the inlet tube. In our case, a tube with a radius of $0.4 \mathrm{~mm}$ caused no decrease of the signal amplitude. Depending on its necessity, one can position several notch filters in line to minimize noise coming with the trace gas flow; one filter section can be sufficient. Implementing such a filter increases the response time of the detector.

\section{BIOLOGICAL APPLICATION: ETHYLENE RELEASE FROM CHERRY TOMATOES}

Ethylene and its role in ripening processes has found substantial attention in the past. Since the gas acts as a hormone, already low concentrations can induce changes in plants. ${ }^{3}$ Ripening, ${ }^{23}$ growth, ${ }^{19}$ seed germination, ${ }^{20,21}$ abscission, and senescence ${ }^{22}$ are influenced by this gas and are studied with laser PA spectroscopy. In the ripening process of cherry tomatoes, the role of ethylene has been studied extensively. It was found that the stem scar was the main channel for gas exchange. ${ }^{25,26}$ The stem scar of the tomato is the spot where the coronet is connected to the plant.

Here we concentrate on the last step in the biosynthesis of ethylene. In this last step, oxygen is necessary to produce $\mathrm{C}_{2} \mathrm{H}_{4}$ from 1-aminocyclopropane-1-carboxylic acid (ACC). ${ }^{24}$ Our interest concerns the delay (at the time scale of seconds) with which ethylene emission from the tomato is changed at the start of anoxic (only $\mathrm{N}_{2}$ flow) periods.

Our experiments were performed on intact detached cherry-tomatoes (Lycopersicon esculentum cherry) in a mature red stage without coronet. A single tomato was positioned inside a specially designed cuvette (Fig. 7). When switching from air to $\mathrm{N}_{2}$ or vice versa $\mathrm{O}_{2}$ was added before or behind the sampling cuvette. Teflon tubing $(0.8 \mathrm{~mm}$ diam, length $1 \mathrm{~m}$ ) was employed to lead the trace gas to the PA cell. Between the tomato and the detector, a $\mathrm{CO}_{2}$ scrubber $(\mathrm{KOH}), 8 \mathrm{~mm}$ diam, length $60 \mathrm{~mm})$ and a cooling trap $(5$ $\mathrm{mm}$ diam, length $60 \mathrm{~mm}$ ) were positioned, the latter to remove water vapor and ethanol. The PA cell was placed intracavity in the $\mathrm{CO}_{2}$ laser. ${ }^{4}$ The measurements were performed at the $\mathrm{C}_{2} \mathrm{H}_{4}$ absorbing laser line $(10 \mathrm{P} 14, \alpha=23.7$ $\mathrm{atm}^{-1} \mathrm{~cm}^{-1}$ ) and at a reference line (10P12).

The observed fast changes in ethylene release were taken as proof that the PA setup was able to follow these type of processes with a time response of a few seconds. In Fig. 8, ethylene release is shown for a cherry tomato from which the coronet had been removed. After an aerobic start, the aerobic conditions were interchanged with anoxic conditions once per minute. During the short anoxic periods, ethylene levels decayed while an increase was observed under re-established aerobic conditions. At $t=237 \mathrm{~min}$, anoxic conditions were 


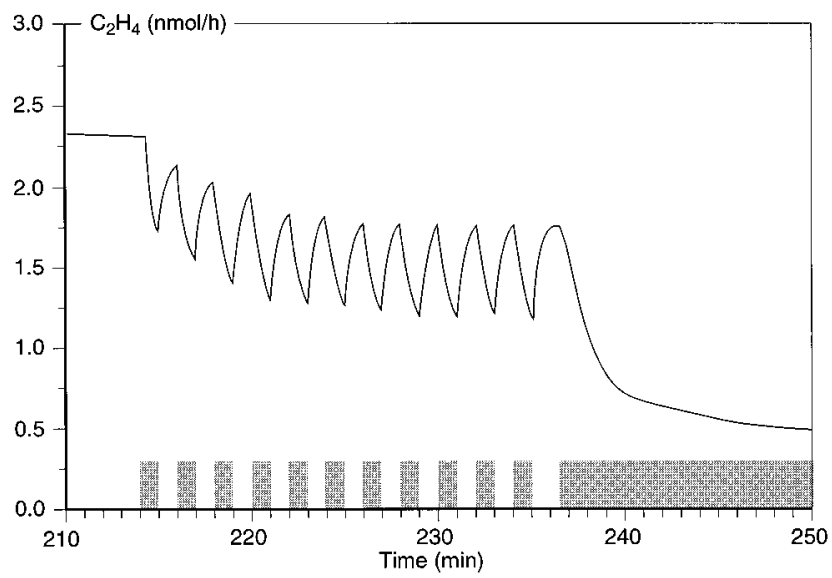

FIG. 8. Fast changing of ethylene emission during alternating aerobic and anaerobic (indicated by a dashed bar) conditions of a ripe tomato.

maintained and the ethylene concentration leveled off toward a low value. This decay allowed to fit an exponential decay function from which a decay time $\tau_{d}=2.1 \pm 0.2 \mathrm{~min}$ could be derived.

After the change from anoxic to aerobic conditions, $\mathrm{O}_{2}$ diffuses into the tomato where it permits ACC oxydase to synthesize ethylene from ACC. At the start, the partial pressure of $\mathrm{O}_{2}$ inside the tomato rises due to diffusion. The storage capacity per unit volume of water (for $\mathrm{O}_{2}$ and ethylene) is ten fold lower than that of air. Additionally, the transport rate of these gases in water is four orders of magnitude lower. To a large extent, the produced ethylene will instantaneously leave the tomato through the air channels along the stem scar. The rate at which $\mathrm{O}_{2}$ flows in and $\mathrm{C}_{2} \mathrm{H}_{4}$ out is, among other factors, determined by the diffusion coefficient, the length of the pathway and the partial pressure difference along the pathway. Furthermore to produce ethylene $\mathrm{O}_{2}$ must reach ACC which is solved in water and then $\mathrm{C}_{2} \mathrm{H}_{4}$ has to diffuse out again. For a light molecule like $\mathrm{O}_{2}$ or $\mathrm{C}_{2} \mathrm{H}_{4}$ to diffuse in water over distances of $1 \mathrm{~mm}$, it takes a diffusion time of $6 \mathrm{~min}$. (over $10 \mathrm{~mm}$ : $10 \mathrm{~h}$ ). Consequently, the observed exponential behavior could stem from diffusion limited processes like the diffusion of ethylene from the bulk of the cherry tomato to the exit at the stem scar or oxygen diffusion inward.

\section{ACKNOWLEDGMENTS}

The authors wish to thank E. M. Mateman for performing the experiments on the cherry tomatoes and Dr. A. Olafsson for critically reading the manuscript.

\section{APPENDIX: INPUT IMPEDANCE OF THE CONDENSOR MICROPHONE}

Here we describe the strongly changed acoustic behavior of the resonator due to influence of a large condensor microphone, its volume in front of the membrane and the small connecting duct to the resonator. To calculate the electrical analog, we assume the acoustic wavelength much larger than

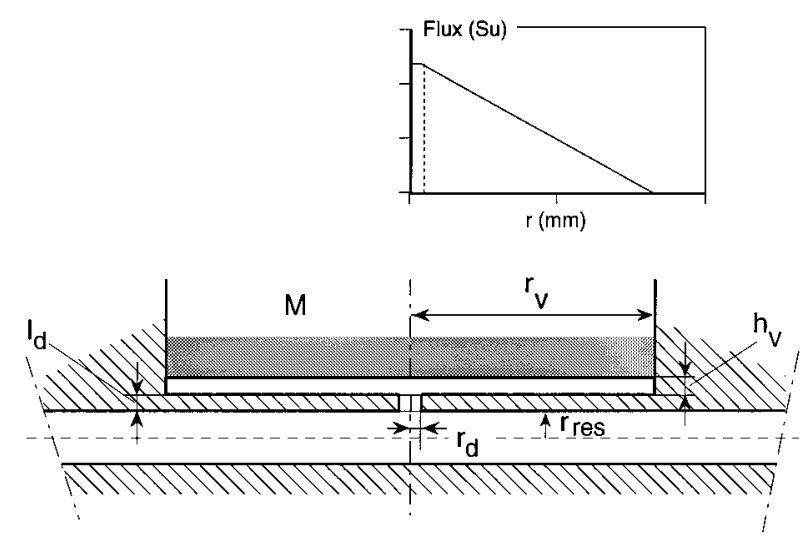

FIG. 9. Flux distribution $S u(r)$ as function of $r$ in the cylinder volume in front of the microphone membrane; $r_{d}$ and $r_{v}$ are the radii of the duct and the cylinder, respectively, $l_{d}$ is the length of the duct and $h_{v}$ the height of the cylinder.

the linear dimensions. Then there will be only heat conduction losses with no viscosity losses, and the volume acts as a capacitor:

$$
C_{v}=\frac{V_{v}}{\rho c^{2}}, \quad G_{v}=\frac{(\gamma-1) \omega S_{v} \delta_{\kappa}}{2 \rho c^{2}}
$$

with $S_{v}$ the inner surface area and $V_{v}$ the internal volume in front of the membrane (Fig. 9). The duct connecting the volume with the resonator possesses a small length and can be described with an inductance and resistance:

$$
L_{d}=\frac{\rho l_{d}}{S_{d}}, \quad R_{d}=\frac{D_{d} l_{d} \rho \omega \delta_{\nu}}{2 S_{d}^{2}}
$$

with $S_{d}$ the cross section, $l_{d}$ the length, and $D_{d}$ the perimeter of the duct. In contrast to the coupled volume, only viscous losses occur. The small capacity term of the duct is neglected. The small duct together with the volume act as a Helmholtz resonator with a resonance frequency and quality factor: ${ }^{10}$

$$
\begin{aligned}
& \omega_{H}=\sqrt{\frac{1}{L_{d} C_{v}}}=\sqrt{\frac{c^{2} S_{d}}{l_{d} V_{v}}}, \\
& Q_{H}=\frac{2 S_{d} V_{v}}{(\gamma-1) S_{v} S_{d} \delta_{\kappa}+V_{v} D_{d} \delta_{\nu}} .
\end{aligned}
$$

Unfortunately, the small volume in front of the microphone does not comply with the assumptions of an ideal Helmholtz resonator. The geometry of the volume deviates strongly from the shape of a sphere and viscous losses must be taken into account. The volume looks like a flat cylinder. From the center of the flat cylinder wall-where the coupling duct enters - a wave spreads out to the edge of the cylinder. A solution for the pressure distribution is given by

$$
p(r, t)=A\left[J_{0}\left(\beta_{r} r\right)+i N_{0}\left(\beta_{r} r\right)\right] e^{i \omega t},
$$

where $J_{0}$ and $N_{0}$ are the zeroth order Bessel and Neumann function. In the case when $\beta_{r} r$ is small, this can be approximated by

$$
p(r, t)=C \ln \left(\beta_{r} r\right) e^{i \omega t} .
$$




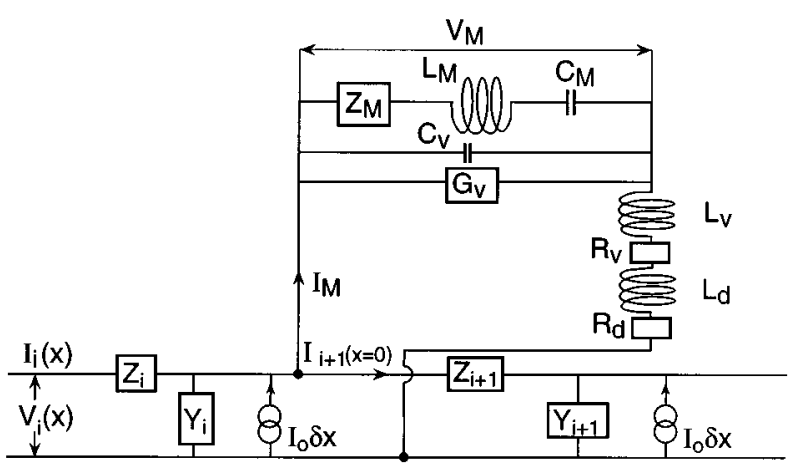

FIG. 10. Analog electric scheme of the condenser microphone (subscript $\mathrm{M})$, with cylinder volume (v) in front of the microphone and connecting duct (d) to the resonator.

The gas flux $U(r)$ is a linearly decreasing function of $r$ (see Fig. 9); the boundary conditions for the gas flux are given by

$$
\begin{aligned}
& 2 \pi r h_{v} u_{v}(r)=0 \quad \text { for } r=r_{v}, \\
& 2 \pi r h_{v} u_{v}(r)=S_{d} U_{d} \text { for } r=r_{d}
\end{aligned}
$$

with $h_{v}$ the height of the cylinder, $u_{v}(r)$ the velocity at radius $r$ in the flat cylinder and $u_{d}$ the velocity in the duct. The first equation takes the zero velocity at the outer diameter of the flat cylinder into account, the second describes the conservation of flux from the connecting duct into the flat cylinder. From the continuity equation in cylindrical coordinates follows:

$$
u_{v}(r)=\frac{S_{d} u_{d}\left(r_{v}^{2}-r^{2}\right)}{S_{v}\left(r_{v}^{2}-r_{d}^{2}\right)}, \quad r \geqslant r_{d}
$$

with $S_{v}=2 \pi r_{v} h_{v}$ the surface area of the cylinder at radius $r$. Based on the kinetic energy considerations, the induction and resistance of the cylinder become:

$$
\begin{aligned}
L_{m} & =\frac{2 E_{\mathrm{kin}}}{\left(S_{d} u_{d}\right)^{2}}=\int_{0}^{r_{v}} \frac{S_{v} \rho u_{v}^{2}(r)}{\left(S_{d} u_{d}\right)^{2}} d r \\
& =\frac{\rho}{4 \pi h_{v}}+\frac{\rho\left[r_{v}^{4}\left\{\ln \left(r_{v} / r_{d}\right)-2 / 3\right\}+r_{d}^{2} r_{v}^{2}-r_{d}^{4} / 3\right]}{2 \pi h_{v}\left(r_{v}^{2}-r_{d}^{2}\right)^{2}}, \\
R_{v} & =\frac{\omega h_{v} \rho\left[r_{v}^{4}\left\{\ln \left(r_{v} / r_{d}\right)-2 / 3\right\}+r_{d}^{2} r_{v}^{2}-r_{d}^{4} / 3\right]}{\pi h_{v}^{2}\left(r_{v}^{2}-r_{d}^{2}\right)^{2}} .
\end{aligned}
$$

So far we have assumed that the walls of the flat cylinder are rigid. In the case of a microphone membrane at one side of the cylinder, the pressure causes a motion of the membrane, which in turn compresses and expands the air layer between the membrane and the back plate of the microphone. This can also be represented by electrical circuit elements. Movement of the membrane increases the kinetic energy and is represented in an inductance $L_{m}\left(=77 \mathrm{~N} \mathrm{~s}^{2} / \mathrm{m}^{5}\right)$. Compression of the air layer and membrane results in an increased potential energy represented by a capacity $C_{m}$ $\left(=2.910^{-12} \mathrm{~m}^{5} / \mathrm{N}\right)$. Viscous losses are represented by a resistance $R_{m}\left(=1.2510^{6} \mathrm{~N} \mathrm{~s} / \mathrm{m}^{5}\right)$. These circuit elements have been calculated for various types of condenser microphone. ${ }^{27}$ For the specific condenser microphone B\&K 4179, they are given by the manufacturer. These additional circuit elements for the membrane and the air layer must be added to the above calculated circuit elements [Eqs. (A2) and (A8)]. The total input impedance of the microphone with volume in front is depicted in Fig. 10.

${ }^{1}$ Air Monitoring by Spectroscopic Techniques, edited by M. W. Sigrist (Wiley, New York, 1994).

${ }^{2}$ Photoacoustic and Photothermal Phenomena III, edited by D. D. Bicanic (Springer, Heidelberg, 1994).

${ }^{3}$ Ethylene in Plant Biology, edited by F. B. Abeles (Academic, New York, 1992).

${ }^{4}$ F. J. M. Harren, F. G. C. Bijnen, J. Reuss, L. A. C. J. Voesenek, and C. W. P. M. Blom, Appl. Phys. B 50, 137 (1990).

${ }^{5}$ R. Gerlach and N. M. Amer, Appl. Phys. 23, 319 (1980).

${ }^{6}$ E. Nodov, Appl. Opt. 17, 1110 (1978).

${ }^{7}$ E. Kritchman, S. Shtrikman, and M. Slatkine, J. Opt. Soc. Am. 68, 1257 (1978).

${ }^{8}$ C. F. Dewey and R. D. Hackett, Appl. Phys. Lett. 23, 633 (1973).

${ }^{9}$ A. Miklos and A. Lorincz, Appl. Phys. B 48, 213 (1989).

${ }^{10}$ S. Bernegger and M. W. Sigrist, Infrared Phys. 30, 375 (1990).

${ }^{11}$ Theoretical Acoustics, edited by P. M. Morse, and K. U. Ingard (McGrawHill, New York, 1968).

${ }^{12}$ T. H. Vansteenkite, F. R. Faxvog, and D. M. Roessler, Appl. Spectrosc. 35, 194 (1981).

${ }^{13}$ S. Bernegger and M. W. Sigrist, Appl. Phys. B 44, 234 (1987).

${ }^{14}$ A. C. Tam, Rev. Mod. Phys. 58, 381 (1986).

${ }^{15}$ F. E. Grigoryan, Sov. Phys. Acoust. 30, 22 (1984).

${ }^{16}$ Gas Encyclopedia, edited by X. Medard (Elsevier, Amsterdam, 1976).

${ }^{17}$ R. J. Brewer, C. W. Bruce, and J. L. Mater, Appl. Opt. 21, 4093 (1982).

${ }^{18}$ F. G. C. Bijnen, F. J. M. Harren, J. H. P. Hackstein, and J. Reuss, Appl. Opt. (in press).

${ }^{19}$ L. A. C. J. Voesenek, M. Banga, R. H. Thier, C. M. Mudde, F. J. M. Harren, G. W. M. Barendse, and C. W. P. M. Blom, Plant Physiol. 103, 783 (1993).

${ }^{20}$ F. J. M. Harren, H. S. M. de Vries, J. Reuss, J. W. J. F. Thuring, G. H. L. Nefkens, and B. Zwanenburg, J. Phys. IV, C 7, 539 (1994).

${ }^{21}$ L. Petruzzelli, F. J. M. Harren, C. Perrone, and J. Reuss, J. Plant Physiol. 145, 83 (1995).

${ }^{22}$ E. J. Woltering, F. J. M. Harren, and H. A. M. Boerrigter, Plant Physiol. 88, 506 (1988).

${ }^{23}$ H. S. M. de Vries, F. J. M. Harren, L. A. C. J. Voesenek, C. W. P. M. Blom, E. J. Woltering, H. C. P. M. van der Valk, and J. Reuss, Plant Physiol. 107, 1371 (1995).

${ }^{24}$ S. F. Yang and N. E. Hoffman, Annu. Rev. Plant Physiol. 35, 155 (1984).

${ }^{25}$ S. P. Burg and E. A. Burg, Physiol. Plant. 18, 870 (1965).

${ }^{26}$ A. C. Cameron and S. F. Yang, Plant Physiol. 70, 21 (1982).

${ }^{27}$ A. J. Zuckerwar, J. Acoust. Soc. Am. 64, 1278 (1978). 